\title{
The Permanence in a Single Species Nonautonomous System with Delays and Feedback Control
}

\author{
Xiaomei Feng and Fengqin Zhang \\ Department of Mathematics, Yuncheng University, Yuncheng 044000, China \\ Correspondence should be addressed to Fengqin Zhang, zhafq@263.net
}

Received 27 October 2009; Accepted 6 January 2010

Academic Editor: Guang Zhang

Copyright (c) 2010 X. Feng and F. Zhang. This is an open access article distributed under the Creative Commons Attribution License, which permits unrestricted use, distribution, and reproduction in any medium, provided the original work is properly cited.

We consider a single species nonautonomous system with delays and feedback control. A general criterion on the permanence for all positive solutions is established. The results show that the feedback control does not influence the permanence of species.

\section{Introduction}

As we well know, a single species without feedback control is very important on mathematical ecology and has been studied in many articles. Many important results on the permanence, extinction, global asymptotical stability, and their special cases of periodic and almost periodic system can be found in [1-6].

However, we note that ecosystem in the real world is continuously disturbed by unpredictable forces which can result in changes in the biological parameters such as survival rates. Of practical interest in ecosystem is the question of whether or not an ecosystem can withstand those unpredictable forces which persist for a finite period of time. In the language of control variables, we call the disturbance functions as control variables. In 1993, Gopalsamy and Weng [7] introduced a feedback control variable into the delay logistic model and discussed the asymptotic behavior of solution in logistic models with feedback controls, in which the control variables satisfy certain differential equation. In the recent years, the population dynamical systems with feedback controls have been studied in many articles, for example, see [8-13] and references cited therein. 
Motivated by the previous works, we focus our attention on the permanence of species for the following single specie non-autonomous systems with delays and feedback control

$$
\begin{gathered}
x^{\prime}(t)=x(t)\left(r(t)-a(t) x(t)-b(t) P\left(x\left(t-\tau_{1}(t)\right)\right)-c(t) Q(u(t-\sigma(t)))\right), \\
u^{\prime}(t)=-d(t) u(t)+e(t) G\left(x\left(t-\tau_{2}(t)\right)\right) .
\end{gathered}
$$

The main purpose of this paper is to establish a new general criterion for the permanence of system (1.1), which is described by integral form and independent of feedback control. The paper is organized as follows. In the next section, we will give some assumptions and useful lemmas. In Section 3, some new sufficient conditions which guarantee the permanence of all positive solutions for system (1.1) are obtained. In the last section, we will give an example to illustrate the conclusions obtained in this paper.

\section{Preliminaries}

In this paper, for system (1.1) we denote that $x(t)$ is the density of the species at time $t, u(t)$ is the control variable, and $(x(t), u(t))=(x(t), u(t))^{T}, r(t), a(t)$ represent the intrinsic growth rate and density-dependent coefficient of the species at time $t$. The function $r(t)$ is bounded continuous defined on $R_{+}=[0, \infty)$; functions $a(t), b(t), c(t), d(t), e(t), \sigma(t)$, and $\tau_{i}(t)(i=1,2)$ are continuous, bounded, and nonnegative defined on $R_{+}$; functions $P(u), Q(u)$, and $G(u)$ are nondecrease defined on $R_{+}$and satisfy $P(0)=0, Q(0)=0$, and $G(0)=0$. Furthermore, there exist positive constants $N, I$, and $L$ such that $|P(u)-P(v)| \leq N|u-v||Q(u)-Q(v)| \leq I|u-v|$, and $|G(u)-G(v)| \leq L|u-v|$ for all $u, v \in R_{+}$.

Throughout this paper, we will introduce the following assumptions:

$\left(\mathrm{H}_{1}\right)$ there exists constant $w>0$ such that

$$
\liminf _{t \rightarrow \infty} \int_{t}^{t+w} r(s) d s>0
$$

$\left(\mathrm{H}_{2}\right)$ there exists constant $\mathcal{\lambda}>0$ such that

$$
\liminf _{t \rightarrow \infty} \int_{t}^{t+\lambda} a(s) d s>0
$$

$\left(\mathrm{H}_{3}\right)$ there exists constant $\gamma>0$ such that

$$
\liminf _{t \rightarrow \infty} \int_{t}^{t+\gamma} d(s) d s>0
$$

In addition, for a function $g(t)$ defined on set $I \subset R$, we denote

$$
g^{L}=\inf _{t \in I} g(t), \quad g^{M}=\sup _{t \in I} g(t) .
$$


Now, we state several lemmas which will be useful in the proving of main results in this paper.

First, we consider the following nonautonomous logistic equation:

$$
x^{\prime}(t)=x(t)(r(t)-a(t) x(t)),
$$

where functions $a(t), r(t)$ are bounded and continuous on $R_{+}$. Furthermore, $a(t) \geq 0$ for all $t \geq 0$. We have the following result which is given in [14] by Teng and Li.

Lemma 2.1. Suppose that assumptions $\left(H_{1}\right)-\left(H_{2}\right)$ hold. Then,

(a) there exist positive constants $m$ and $M$ such that

$$
m \leq \liminf _{t \rightarrow \infty} x(t) \leq \limsup _{t \rightarrow \infty} x(t) \leq M
$$

for any positive solution $x(t)$ of (2.5);

(b) $\lim _{t \rightarrow \infty}\left(x^{(1)}(t)-x^{(2)}(t)\right)=0$ for any two positive solutions $x^{(1)}(t)$ and $x^{(2)}(t)$ of $(2.5)$.

Further, we consider the following nonautonomous linear equation:

$$
u^{\prime}(t)=r(t)-d(t) u(t)
$$

where functions $r(t)$ and $d(t)$ are bounded continuous defined on $R_{+}$, and $r(t) \geq 0$ for all $t \geq 0$. We have the following result.

Lemma 2.2. Suppose that assumption $\left(\mathrm{H}_{3}\right)$ holds. Then,

(a) there exists a positive constant $U$ such that $\lim _{\sup } \operatorname{su}_{t \rightarrow \infty} u(t) \leq U$ for any positive solution $u(t)$ of $(2.7)$;

(b) $\lim _{t \rightarrow \infty}\left(u^{(1)}(t)-u^{(2)}(t)\right)=0$ for any two positive solutions $u^{(1)}(t)$ and $u^{(2)}(t)$ of $(2.7)$.

The proof of Lemma 2.2 is very simple by making a transformation with $u(t)=1 / x(t)$. This produces the calculations $u^{\prime}(t)=-\left(1 / x^{2}(t)\right) x^{\prime}(t)$ and $x^{\prime}(t)=x(t)(e(t)-r(t) x(t))$. Then, according to Lemma 2.1 we can obtain Lemma 2.2.

Lemma 2.3. Suppose that assumption $\left(H_{3}\right)$ holds. Then for any constants $\epsilon>0$ and $M>0$ there exist constants $\delta=\delta(\epsilon)>0$ and $T=T(M)>0$ such that for any $t_{0} \in R_{+}$and $u_{0} \in R$ with $\left|u_{0}\right| \leq M$, when $|r(t)|<\delta$ for all $t \geq t_{0}$, one has

$$
\left|u\left(t, t_{0}, u_{0}\right)\right|<\epsilon \quad \forall t \geq t_{0}+T,
$$

where $u\left(t, t_{0}, u_{0}\right)$ is the solution of (2.7) with initial condition $u\left(t_{0}\right)=u_{0}$.

The proof of Lemma 2.3 can be found as Lemma 2.4 in [12] by Wang et al. 


\section{Main Results}

Let $\tau=\sup \left\{\tau_{1}(t), \tau_{2}(t), \sigma(t): t \geq t_{0}\right\}$. We define $C[-\tau, 0]$ the Banach space of bounded continuous functions $\phi:[-\tau, 0] \rightarrow R$ with the supremum norm defined by $\|\phi\|_{\mathcal{C}}=$ $\sup _{-\tau \leq s \leq 0}|\phi(s)|$. By the fundamental theory of functional differential equation, we know that for any $(\phi, \psi) \in C[-\tau, 0] \times C[-\tau, 0]$, system (1.1) has a unique solution $X(t, \phi, \psi)=$ $\{x(t, \phi, \psi), u(t, \phi, \psi)\}$ satisfying the initial condition $X_{t_{0}}=(\phi, \psi)$.

Motivated by the biological background of system (1.1), in this paper we are only concerned with positive solutions of system (1.1). It is not difficult to see that the solution $\{x(t, \phi, \psi), u(t, \phi, \psi)\}$ of system (1.1) is positive, if the initial functions satisfy $\phi(s) \geq 0$ and $\phi(0)>0 ; \psi(s) \geq 0$ and $\psi(0)>0$ for all $s \in[-\tau, 0]$.

Theorem 3.1. Suppose that assumptions $\left(H_{1}\right)-\left(H_{3}\right)$ hold. Then there exists constant $M>0$ such that

$$
\limsup _{t \rightarrow \infty} x(t)<M, \quad \limsup _{t \rightarrow \infty} u(t)<M,
$$

for any positive solutions $(x(t), u(t))$ of system (1.1).

Proof. Let $\{x(t), u(t)\}$ be any positive solution of system (1.1). Since

$$
\frac{d x(t)}{d t} \leq x(t)(r(t)-a(t) x(t))
$$

for all $t \geq t_{0}$, where $t_{0}$ is the initial time.

Consider the following auxiliary equation:

$$
\frac{d y(t)}{d t}=y(t)(r(t)-a(t) y(t))
$$

from assumptions $\left(\mathrm{H}_{1}\right)$ and $\left(\mathrm{H}_{2}\right)$ and according to Lemma 2.1, there exists constant $M_{1}>0$ such that

$$
\limsup _{t \rightarrow \infty} y(t) \leq M_{1}
$$

for the solution $y(t)$ of (3.3) with initial condition $y\left(t_{0}\right)=x\left(t_{0}\right)$. By the comparison theorem, we have

$$
x(t) \leq y(t) \quad \forall t \geq t_{0} .
$$

From this, we further obtain

$$
\limsup _{t \rightarrow \infty} x(t) \leq M_{1}
$$


Then, we obtain that for any constant $\varepsilon>0$, there exists constant $T>t_{0}$ such that

$$
x(t)<M_{1}+\varepsilon \quad \forall t \geq T .
$$

From the second equation of the system (1.1), we have

$$
\frac{d u(t)}{d t} \leq-d(t) u(t)+L e(t) x\left(t-\tau_{2}(t)\right)
$$

Hence, we further have

$$
\frac{d u(t)}{d t} \leq-d(t) u(t)+L e(t)\left(M_{1}+\varepsilon\right)
$$

for all $t \geq T+\tau$. Consider the following auxiliary equation:

$$
\frac{d v(t)}{d t}=-d(t) v(t)+L e(t)\left(M_{1}+\varepsilon\right)
$$

from assumption $\left(\mathrm{H}_{3}\right)$ and the conclusions of Lemma 2.2, we can get constant $M_{2}>0$ such that for any $\varepsilon>0$ there exists constant $T_{1} \geq T+\tau$ such that

$$
v(t)<M_{2}+\varepsilon
$$

for the solution $v(t)$ of (3.10) with initial condition $v\left(T_{1}\right)=u\left(T_{1}\right)$. By the comparison theorem, we have

$$
u(t) \leq v(t) \quad \forall t \geq T_{1}
$$

Hence, we further obtain

$$
\limsup _{t \rightarrow \infty} u(t)<M_{2}
$$

Choose the constant $M=\max \left\{M_{1}, M_{2}\right\}$, then we finally obtain

$$
\limsup _{t \rightarrow \infty} x(t)<M, \quad \limsup _{t \rightarrow \infty} u(t)<M
$$

This completes the proof.

Theorem 3.2. Suppose that assumptions $\left(H_{1}\right)-\left(H_{3}\right)$ hold. Then there exists constant $l>0$ such that

$$
\liminf _{t \rightarrow \infty} x(t)>l
$$

for any positive solutions $(x(t), u(t))$ of system (1.1). 
Proof. According to assumption $\left(\mathrm{H}_{1}\right)$, we can choose constants $\varepsilon_{0}>0$ and $T_{0}>0$ such that for all $t \geq T_{0}$, we have

$$
\int_{t}^{t+w}\left(r(s)-a(t) \varepsilon_{0}-b(t) \varepsilon_{0}-c(t) \varepsilon_{0}\right) d s>\varepsilon_{0}
$$

We consider the following equation:

$$
\frac{d u(t)}{d t}=-d(t) u(t)+e(t) \alpha_{0}
$$

where $\alpha_{0}$ is parameter. By Lemma 2.3, for given $\varepsilon_{0}>0$ and positive constant $M>0$, there exist constants $\delta_{0}=\delta_{0}\left(\varepsilon_{0}\right)>0$ and $T^{*}=T^{*}(M)>0$ such that for any $t_{0} \in R_{+}$and $0 \leq u_{0} \leq M$, when $\alpha_{0} e(t)<\delta_{0}$ for all $t \geq t_{0}$, we have

$$
0 \leq u\left(t, t_{0}, u_{0}\right)<\varepsilon_{0} \quad \forall t \geq t_{0}+T^{*},
$$

where $u\left(t, t_{0}, u_{0}\right)$ is the solution of (3.17) with initial condition $u\left(t_{0}\right)=u_{0}$.

Let $\alpha_{0} \leq \min \left\{\varepsilon_{0}, \delta_{0} /\left(e^{M}+1\right)\right\}$ such that for all $t \geq T_{0}$

$$
\int_{t}^{t+w}\left(r(s)-a(t) \frac{\alpha_{0}}{L}-b(t) \frac{N \alpha_{0}}{L}-c(t) I \varepsilon_{0}\right) d s>\alpha_{0} .
$$

We first prove that

$$
\limsup _{t \rightarrow \infty} x(t) \geq \frac{\alpha_{0}}{L}
$$

In fact, if (3.20) is not true, then there exists a positive solution $\{x(t), u(t)\}$ of system (1.1), a constant $T_{1}>T_{0}$ such that $x(t)<\alpha_{0} / L$ for all $t \geq T_{1}$. Then for all $t \geq T_{1}+\tau$, we have

$$
\frac{d u(t)}{d t} \leq-d(t) u(t)+e(t) \alpha_{0}
$$

Let $v(t)$ be the solution of (3.17) with initial condition $v\left(T_{1}+\tau\right)=u\left(T_{1}+\tau\right)$, by the comparison theorem, we have

$$
u(t) \leq v(t) \quad \forall t \geq T_{1}+\tau .
$$

In (3.18), we choose $t_{0}=T_{1}+\tau$ and $u_{0}=u\left(T_{1}+\tau\right)$, since $\alpha_{0} e(t)<\delta_{0}$ for all $t \geq T_{1}+\tau$, we obtain

$$
v(t)=v\left(t, T_{1}+\tau, u\left(T_{1}+\tau\right)\right)<\varepsilon_{0} \quad \forall t \geq T_{1}+\tau+T^{*} .
$$

Hence, from (3.22) we further obtain

$$
u(t)<\varepsilon_{0} \quad \forall t \geq T_{1}+\tau+T^{*} .
$$


Considering the first equation (1.1), for any $t \geq T_{1}+T^{*}+2 \tau$, we have

$$
\frac{d x(t)}{d t} \geq x(t)\left(r(s)-a(t) \frac{\alpha_{0}}{L}-b(t) \frac{N \alpha_{0}}{L}-c(t) I \varepsilon_{0}\right) .
$$

Integrating (3.25) form $T_{1}+T^{*}+2 \tau$ to $t \geq T_{1}+T^{*}+2 \tau$, we have

$$
x(t) \geq x\left(T_{1}+T^{*}+2 \tau\right) \exp \int_{T_{1}+T^{*}+2 \tau}^{t}\left(r(s)-a(t) \frac{\alpha_{0}}{L}-b(t) \frac{N \alpha_{0}}{L}-c(t) I \varepsilon_{0}\right) d s .
$$

Obviously, from (3.19) and (3.26), we obtain $x(t) \rightarrow \infty$ as $t \rightarrow \infty$, which leads to a contradiction.

Now, we prove the conclusion of Theorem 3.2. In fact, if it is not true, then there exists a sequence $\left\{\phi^{(m)}, \psi^{(m)}\right\}$ of initial functions such that, for the solution $\left\{x\left(t, \phi^{(m)}\right.\right.$, $\left.\left.\psi^{(m)}\right), u\left(t, \phi^{(m)}, \psi^{(m)}\right)\right\}$ of the system (1.1)

$$
\liminf _{t \rightarrow \infty} x\left(t, \phi^{(m)}, \psi^{(m)}\right)<\frac{\beta}{m^{2}} \quad \forall m=1,2, \ldots
$$

where $\beta=\alpha_{0} / L$ and $\left\{x\left(t, \phi^{(m)}, \psi^{(m)}\right), u\left(t, \phi^{(m)}, \psi^{(m)}\right)\right\}$ satisfies the initial condition

$$
x(s)=\phi^{(m)}(s), \quad u(s)=\psi^{(m)}(s) \quad \forall t \in[-\tau, 0] .
$$

From (3.20) and (3.27), for every $m$ there are two time sequences $\left\{s_{q}^{(m)}\right\}$ and $\left\{t_{q}^{(m)}\right\}$, satisfying $0<s_{1}^{(m)}<t_{1}^{(m)}<s_{2}^{(m)}<t_{2}^{(m)}<\cdots<s_{q}^{(m)}<t_{q}^{(m)}<\cdots$ and $\lim _{q \rightarrow \infty} s_{q}^{(m)}=\infty$, such that

$$
\begin{gathered}
x\left(s_{q}^{(m)}, \phi^{(m)}, \psi^{(m)}\right)=\frac{\beta}{m}, \quad x\left(t_{q}^{(m)}, \phi^{(m)}, \psi^{(m)}\right)=\frac{\beta}{m^{2}} \\
\frac{\beta}{m^{2}} \leq x\left(t, \phi^{(m)}, \psi^{(m)}\right) \leq \frac{\beta}{m} \quad \forall t \in\left(s_{q}^{(m)}, t_{q}^{(m)}\right) .
\end{gathered}
$$

From Theorem 3.1, we can choose a positive constant $T^{(m)}$ such that $x\left(t, \phi^{(m)}, \psi^{(m)}\right)<$ $M$, and $u\left(t, \phi^{(m)}, \psi^{(m)}\right)<M$ for all $t>T^{(m)}$. Further, there is an integer $K_{1}^{(m)}>0$ such that $s_{q}^{(m)}>T^{(m)}+\tau$ for all $q \geq K_{1}^{(m)}$. Let $q \geq K_{1}^{(m)}$, for any $t \in\left(s_{q}^{(m)}, t_{q}^{(m)}\right)$ we have

$$
\begin{aligned}
\frac{d x\left(t, \phi^{(m)}, \psi^{(m)}\right)}{d t} & \geq x\left(t, \phi^{(m)}, \psi^{(m)}\right)[r(t)-a(t) M-b(t) N M-c(t) I M] \\
& \geq-\gamma x\left(t, \phi^{(m)}, \psi^{(m)}\right),
\end{aligned}
$$


where $\gamma=\sup _{t \in R_{+}}\{|r(t)|+a(t) M+b(t) N M+c(t) I M\}$. Integrating the above inequality from $s_{q}^{(m)}$ to $t_{q}^{(m)}$, we further have

$$
\begin{aligned}
\frac{\beta}{m^{2}} & =x\left(t_{q}^{(m)}, \phi^{(m)}, \psi^{(m)}\right) \geq x\left(s_{q}^{(m)}, \phi^{(m)}, \psi^{(m)}\right) \exp \left[-\gamma\left(t_{q}^{(m)}-s_{q}^{(m)}\right)\right] \\
& =\frac{\beta}{m} \exp \left[-\gamma\left(t_{q}^{(m)}-s_{q}^{(m)}\right)\right] .
\end{aligned}
$$

Consequently,

$$
t_{q}^{(m)}-s_{q}^{(m)} \geq \frac{\ln m}{r} \quad \forall q \geq K_{1}^{(m)}, m=1,2, \ldots
$$

We can choose a large enough $N_{0}$ such that

$$
t_{q}^{(m)}-s_{q}^{(m)} \geq T^{*}+\tau+w \quad \forall m \geq N_{0}, q \geq K_{1}^{(m)}
$$

For any $m \geq N_{0}, q \geq K_{1}^{(m)}$ and $t \in\left[s_{q}^{(m)}+\tau, t_{q}^{(m)}\right]$, from (3.30) we can obtain

$$
\begin{aligned}
\frac{d u\left(t, \phi^{(m)}, \psi^{(m)}\right)}{d t} & =-d(t) u\left(t, \phi^{(m)}, \psi^{(m)}\right)+e(t) L x\left(\left(t-\tau_{2}\right), \phi^{(m)}, \psi^{(m)}\right) \\
& \leq-d(t) u\left(t, \phi^{(m)}, \psi^{(m)}\right)+e(t) L \frac{\beta}{m} \\
& \leq-d(t) u\left(t, \phi^{(m)}, \psi^{(m)}\right)+e(t) \alpha_{0} .
\end{aligned}
$$

Assume that $\tilde{u}(t)$ is the solution of (3.17) with the initial condition $\widetilde{u}\left(s_{q}^{(m)}+\tau\right)=u\left(s_{q}^{(m)}+\right.$ $\tau)$, then we have

$$
u\left(t, \phi^{(m)}, \psi^{(m)}\right) \leq \widetilde{u}(t) \quad \forall t \in\left[s_{q}^{(m)}+\tau, t_{q}^{(m)}\right], m \geq N_{0}, q \geq K_{1}^{(m)} .
$$

In (3.18), we choose $t_{0}=s_{q}^{(m)}+\tau$ and $u_{0}=u\left(s_{q}^{(m)}+\tau\right)$, since $\alpha_{0} e(t)<\delta_{0}$ for all $t \geq s_{q}^{(m)}+\tau$, we have

$$
\tilde{u}(t)=\tilde{u}\left(t, s_{q}^{(m)}+\tau, u\left(s_{q}^{(m)}+\tau\right)\right)<\varepsilon_{0}
$$

for all $t \in\left[s_{q}^{(m)}+\tau+T^{*}, t_{q}^{(m)}\right]$. Using the comparison theorem it follows that

$$
u\left(t, \phi^{(m)}, \psi^{(m)}\right)<\varepsilon_{0}
$$

for all $t \in\left[s_{q}^{(m)}+\tau+T^{*}, t_{q}^{(m)}\right], q \geq K_{1}^{(m)}$ and $m \geq N_{0}$. 

follows

So, for any $m \geq N_{0}, q \geq K_{1}^{(m)}$ and $t \in\left[s_{q}^{(m)}+\tau+T^{*}, t_{q}^{(m)}\right]$, from (3.30) and (3.38), it

$$
\begin{aligned}
\frac{d x\left(t, \phi^{(m)}, \psi^{(m)}\right)}{d t}= & x\left(t, \phi^{(m)}, \psi^{(m)}\right)\left(r(t)-a(t) x\left(t, \phi^{(m)}, \psi^{(m)}\right)\right. \\
& \left.-b(t) N\left(x\left(t-\tau_{1}(t), \phi^{(m)}, \psi^{(m)}\right)\right)-c(t) I\left(u\left(t-\sigma(t), \phi^{(m)}, \psi^{(m)}\right)\right)\right) \\
\geq & x\left(t, \phi^{(m)}, \psi^{(m)}\right)\left(r(t)-a(t) \frac{\alpha_{0}}{L}-b(t) \frac{N \alpha_{0}}{L}-c(t) I \varepsilon_{0}\right) .
\end{aligned}
$$

Integrating the above inequality from $t_{q}^{(m)}-\omega$ to $t_{q}^{(m)}$, then from (3.19), (3.29), and (3.30), we obtain

$$
\begin{aligned}
\frac{\beta}{m^{2}} & =x\left(t_{q}^{(m)}, \phi^{(m)}, \psi^{(m)}\right) \\
& \geq x\left(t_{q}^{(m)}-\omega, \phi^{(m)}, \psi^{(m)}\right) \exp \int_{t_{q}^{(m)}-\omega}^{t_{q}^{(m)}}\left(r(t)-a(t) \frac{\alpha_{0}}{L}-b(t) \frac{N \alpha_{0}}{L}-c(t) I \varepsilon_{0}\right) d t \\
& \geq \frac{\beta}{m^{2}} \exp \left(\alpha_{0}\right)>\frac{\beta}{m^{2}}
\end{aligned}
$$

which leads to a contradiction. Therefore, this contradiction shows that there exists constant $l>0$ such that

$$
\liminf _{t \rightarrow \infty} x(t)>l,
$$

for any positive solutions $(x(t), u(t))$ of system (1.1). This completes the proof.

Remark 3.3. In Theorem 3.2, we note that $\left(\mathrm{H}_{1}\right)-\left(\mathrm{H}_{3}\right)$ decided by (1.1), which is independent of the feedback controls. So, the feedback controls have no influence on the permanence of system (1.1). That is, there is the permanence of the species as long as feedback controls (human activities) should be kept in a certain range. In the range, the permanence of the species will not be influenced by the controls.

\section{An Example}

In this section we will give an example to illustrate the conclusion obtained in the above section. We will consider the following single species system with delays and feedback control:

$$
\begin{gathered}
x^{\prime}(t)=x(t)\left(r(t)-a(t) x(t)-b(t) P\left(x\left(t-\tau_{1}(t)\right)\right)-c(t) Q(u(t-\sigma(t)))\right), \\
u^{\prime}(t)=-d(t) u(t)+e(t) G\left(x\left(t-\tau_{2}(t)\right)\right),
\end{gathered}
$$


where we have

$$
\begin{array}{cr}
r(t)=\frac{1}{2}+\sin t, \quad a(t)=1+\cos 2 t, \quad b(t)=3+\cos ^{2} t \\
c(t)=\frac{1}{4}+\cos ^{2} t, \quad d(t)=2+\sin 2 t, \quad e(t)=\frac{1}{3}+\frac{1}{3} \cos ^{2} t \\
P(t)=Q(t)=G(t)=t .
\end{array}
$$

Obviously, $a(t), b(t), c(t), d(t), e(t)$ are continuous, bounded, and nonnegative defined on $R_{+}$; functions $P(t), Q(t)$ and $G(t)$ are nondecrease defined on $R_{+}$and satisfy $P(0)=0, Q(0)=0$ and $G(0)=0$. Choose the constants $\omega, \lambda, \gamma: \omega=\lambda=\gamma=2 \pi$, then we easily obtain

$$
\liminf _{t \rightarrow \infty} \int_{t}^{t+2 \pi} r(s) d s>0, \quad \liminf _{t \rightarrow \infty} \int_{t}^{t+2 \pi} a(s) d s>0, \quad \liminf _{t \rightarrow \infty} \int_{t}^{t+2 \pi} d(s) d s>0 .
$$

Therefore, assumptions $\left(\mathrm{H}_{1}\right)-\left(\mathrm{H}_{3}\right)$ hold, we obtain that the species $x(t)$ is persistent.

\section{Acknowledgments}

This work is supported by the Sciences Foundation of Shanxi (2009011005-3) and the Major Subject Foundation of Shanxi.

\section{References}

[1] B. G. Zhang and K. Gopalsamy, "Global attractivity and oscillations in a periodic delay-logistic equation," Journal of Mathematical Analysis and Applications, vol. 150, no. 1, pp. 274-283, 1990.

[2] R. Yuan and J. Hong, "The existence of almost periodic solution of a population equation with delay," Applicable Analysis, vol. 61, no. 1-2, pp. 45-52, 1996.

[3] Z. Teng, "Permanence and stability in non-autonomous logistic systems with infinite delay," Dynamical Systems, vol. 17, no. 3, pp. 187-202, 2002.

[4] Z. Teng, "Persistence and stability in general nonautonomous single-species Kolmogorov systems with delays," Nonlinear Analysis: Real World Applications, vol. 8, no. 1, pp. 230-248, 2007.

[5] H. I. Freedman and J. H. Wu, "Periodic solutions of single-species models with periodic delay," SIAM Journal on Mathematical Analysis, vol. 23, no. 3, pp. 689-701, 1992.

[6] G. Seifert, "On a delay-differential equation for single specie population variations," Nonlinear Analysis: Theory, Methods \& Applications, vol. 11, no. 9, pp. 1051-1059, 1987.

[7] K. Gopalsamy and P. X. Weng, "Feedback regulation of logistic growth," International Journal of Mathematics and Mathematical Sciences, vol. 16, no. 1, pp. 177-192, 1993.

[8] F. Chen, "Positive periodic solutions of neutral Lotka-Volterra system with feedback control," Applied Mathematics and Computation, vol. 162, no. 3, pp. 1279-1302, 2005.

[9] H.-F. Huo and W.-T. Li, "Positive periodic solutions of a class of delay differential system with feedback control," Applied Mathematics and Computation, vol. 148, no. 1, pp. 35-46, 2004.

[10] P. Weng, "Existence and global stability of positive periodic solution of periodic integrodifferential systems with feedback controls," Computers \& Mathematics with Applications, vol. 40, no. 6-7, pp. 747$759,2000$.

[11] F. Yin and Y. Li, "Positive periodic solutions of a single species model with feed-back regulation and distributed time delay," Applied Mathematics and Computation, vol. 153, pp. 475-484, 2004.

[12] K. Wang, Z. Teng, and H. Jiang, "On the permanence for $n$-species non-autononous Lotka-Volterra competitive system with infinite delays and feed-back controls," International Joural of Biomathematics, vol. 1, pp. 29-43, 2008. 
[13] F. Chen, J. Yang, L. Chen, and X. Xie, "On a mutualism model with feedback controls," Applied Mathematics and Computation, vol. 214, no. 2, pp. 581-587, 2009.

[14] Z. Teng and Z. Li, "Permanence and asymptotic behavior of the $N$-species nonautonomous LotkaVolterra competitive systems," Computers E Mathematics with Applications, vol. 39, no. 7-8, pp. 107-116, 2000. 


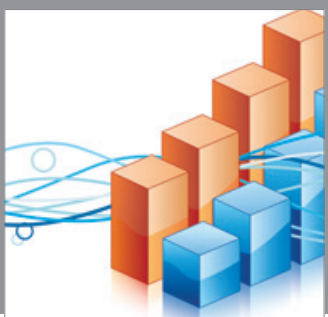

Advances in

Operations Research

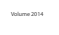

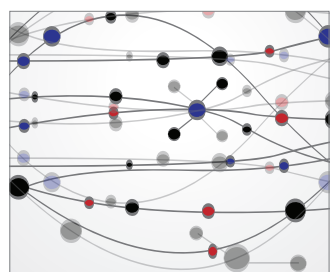

\section{The Scientific} World Journal
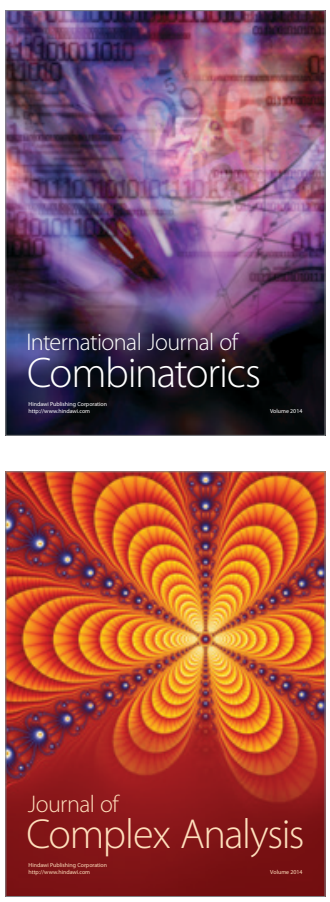

International Journal of

Mathematics and

Mathematical

Sciences
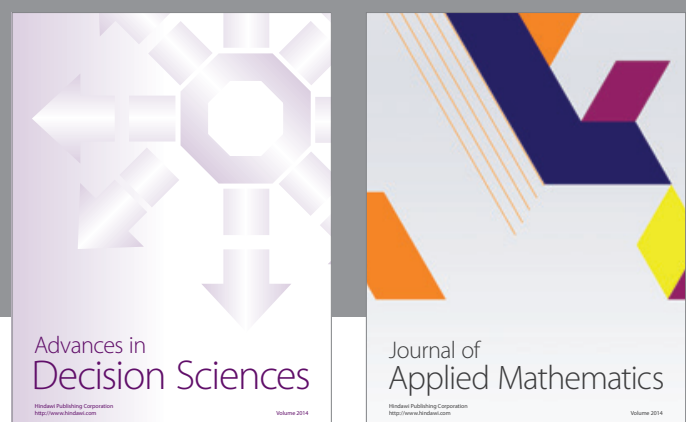

Journal of

Applied Mathematics
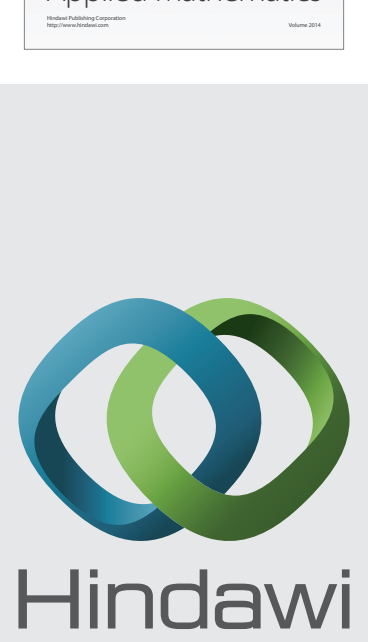

Submit your manuscripts at http://www.hindawi.com
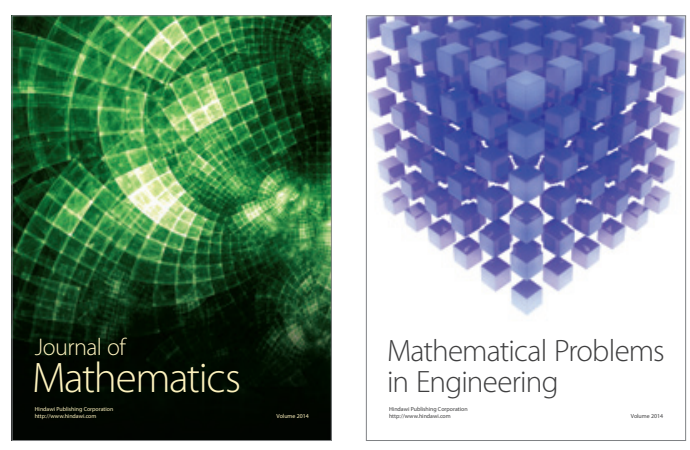

Mathematical Problems in Engineering
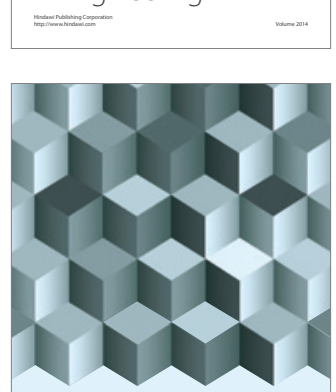

Journal of

Function Spaces
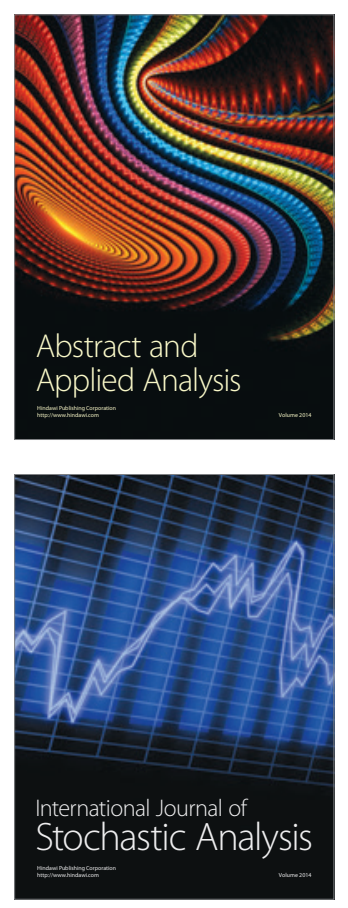

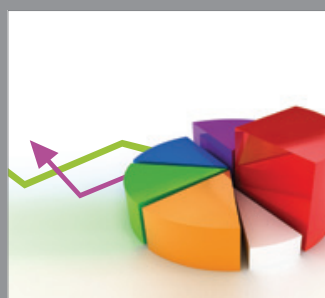

ournal of

Probability and Statistics

Promensencen
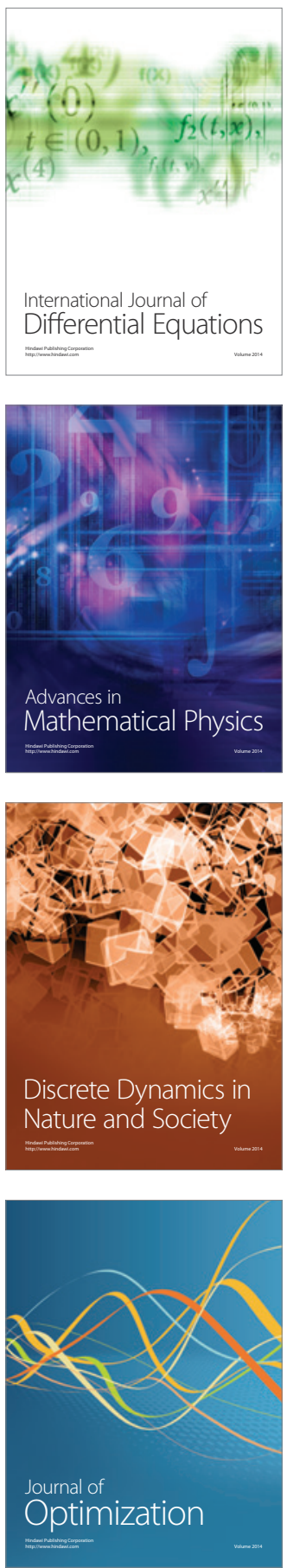